\title{
Pass-by noise synthesis from transfer path analysis using IIR filters
}

\author{
Mansour Alkmim ${ }^{1,2}$, Fabio Bianciardi ${ }^{1}$, Guillaume Vandernoot ${ }^{3}$, Laurent De \\ Ryck $^{1}$, Jacques Cuenca ${ }^{1}$, and Karl Janssens ${ }^{1}$ \\ 1 Siemens Digital Industries Software, Interleuvenlaan 68, B-3001 Leuven, Belgium \\ mansour . alkmim@siemens.com \\ 2 KU Leuven, Department of Mechanical Engineering, Celestijnenlaan 300 B, \\ B-3001, Heverlee, Belgium \\ 3 Siemens Digital Industries Software, 150 avenue de la Republique, 92320, Chatillon, \\ France
}

\begin{abstract}
Component-based Transfer Path Analysis (TPA) has been developed to predict the exterior sound level contribution from noisegenerating components upfront in the design cycle without having to physically integrate them into the vehicle. Virtual pass-by noise predictions for different vehicle variants can be reached by combining the characterization of each component source with simulated vehicle Noise Transfer Functions (NTF). The objective of this work is to implement the technology in a time-domain framework to enable listening tests. The time-varying filters obtained from measured noise transfer functions are implemented as Infinite Impulse Response (IIR) filters. The technique is validated on an automotive example.
\end{abstract}

Keywords: Transfer path analysis, Sound synthesis, Pass-by noise

\section{Introduction}

Pass-By Noise (PBN) engineering is a well-defined procedure in the development process of vehicles. Automotive original equipment manufacturers (OEMs) and component suppliers are looking into techniques that allow setting realistic noise and vibration harshness (NVH) targets for the contributing noise sources and, by doing so, frontloading the pass-by noise performance into the design process. More specifically, the indoor PBN test is becoming an attractive alternative to the exterior PBN due to the easy reproducibility and significantly reduced constraints due to changing environmental conditions. Although the PBN sound levels can be obtained by energetic approach, the current solutions rely on mixing and interpolation techniques [1] which does not provide satisfactory virtual PBN audio signal. Audio synthesis for sound quality assessment has increased in importance due to the electrification of vehicles and for sound quality assessment using psychoacoustic metrics.

The objective of this paper is to develop and implement a time-domain TPA synthesis technique. The sound synthesis of a pass-by run with inclusion of the 
Doppler effect and accurate sound pressure levels is conducted by representing the measured transfer functions as time-varying IIR filters that are updated at sampling-rate speed. The methodology is based on the TPA-ASQ (Airborne Source Quantification) method, which allows to separate the airborne noise sources of a vehicle and evaluate their individual contribution to the overall pass-by noise level [2-4]. The advantages of implementing an IIR filter design are the provided flexibility, efficiency in implementation and the possibility of real time audio applications. The main underlying challenge of the sound synthesis is to obtain a computationally efficient procedure and click-free (no artifacts) solution.

\section{IIR filter design}

The IIR filter design procedure is summarized in Figure 1. The first step consists in obtaining the noise transfer functions (NTF) at discretized target microphone positions (refer to Fig. 2(b)). The second step consists of the decomposition of the NTFs into a minimum-phase and all-pass filter [5]. The minimum phase magnitude is employed in the IIR filter design while the all-pass phase provides the time delay information which can be used to implement the Doppler effect.

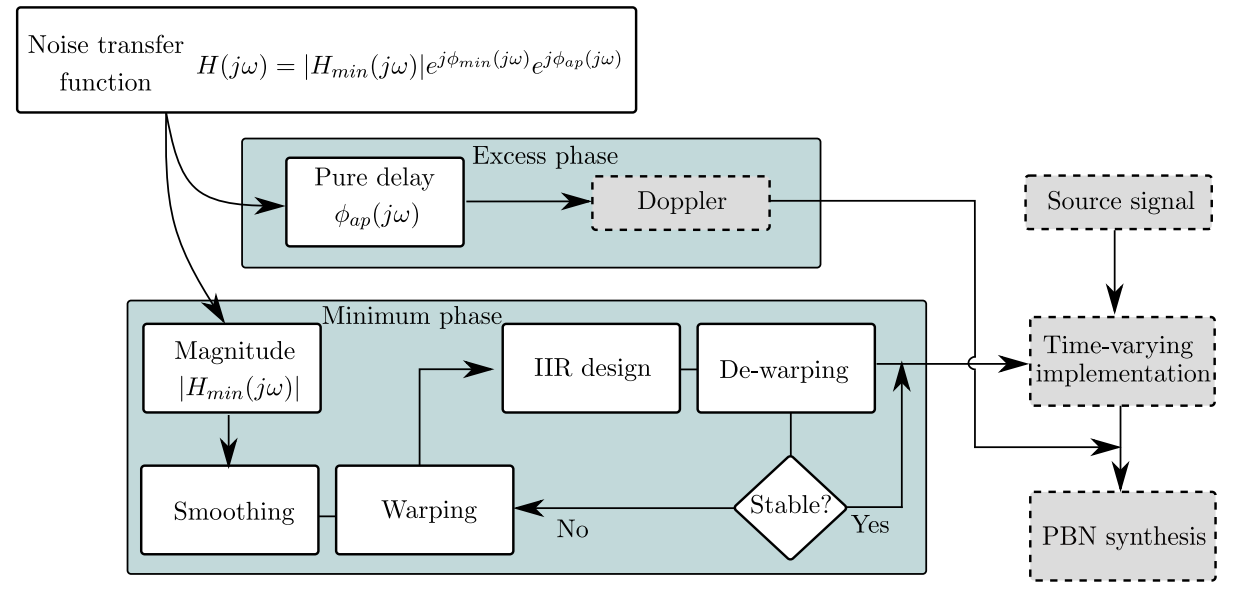

Fig. 1: Pass-by noise synthesis diagram.

\subsection{Transfer-Path-Analysis (TPA) and Airborne Source Quantification (ASQ)}

TPA is a source-transfer-path technique to assess the contributions of multiple sources in a system towards specific targets, for instance the driver's ears in a car. The process exploits the availability of transfer functions between source points and measurement (indicator) points. In the pass-by noise context, targets 
appear as a linear microphone array in the far-field; and sources are airborne, radiating outwards from e.g. the powertrain, the tailpipe, front and rear tires. The set of NTFs between each source and target points represents changes in propagation distance, directivity and angle of incidence.

The ASQ method aims at identifying and separating the airborne noise sources from each component. By assuming that the noise-producing components can be represented by a set of monopole sources, the operational acoustic loads can be identified from independent component measurements by means of inverse techniques. Two types of ASQ are commonly employed, linear phasebased pressure inversion method and power-based energetic approach [6]. While the linear approach accounts for the source phase information, the energetic approach assumes that all sources are uncorrelated.

\subsection{Pre-processing the measured noise transfer functions (NTF)}

Two properties are exploited to reduce the computational cost of the sound synthesis while maintaining the main audio cues [5]. The first property is the pole-zero filters, which states that every stable filter can be decomposed into minimum phase and all-pass filters associated in series. The second property is that all-pass filters can be approximated by pure delays. This property is used to estimate the time delay from the sources to the target microphones. The decomposition of the NTF is given by [5]

$$
H(j \omega)=\left|H_{\min }(j \omega) H_{a p}(j \omega)\right| e^{j \phi_{\min }(j \omega)} e^{j \phi_{a p}(j \omega)},
$$

where $\phi_{\min }(j \omega)$ denotes the minimum phase, $\phi_{a p}(j \omega)$ denotes the excess phase, $\left|H_{\min }(j \omega)\right|$ is the minimum phase magnitude and the $\left|H_{a p}(j \omega)\right|=1$ is the allpass magnitude.

The phase and magnitude of a minimum phase system are uniquely related by the inverse Hilbert transform $\phi_{\min }(j \omega)=\mathscr{H}^{-1}\left\{\ln \left(-\left|H_{\min }(j \omega)\right|\right)\right\}$. Two additional operations, the smoothing and warping are performed to improve the filter fitting at low frequencies. The smoothing consists of a convolution of the NTF with a frequency-dependent Hann window. The warping has the effect of resampling the NTF by replacing in the Z-domain the unit delay $z^{-1}$ by an all-pass filter. Then, the IIR filter is least-square fitted on the warped NTF magnitude employing the Yule-Walker algorithm [5]. Lastly, the resultant filter is de-warped in frequency domain.

\subsection{Implementation of time-varying IIR filter coefficients}

the IIR filter with time varying coefficients is implemented by solving the difference equation described in Eq.(2), which is also known as direct-form I [7]. For a general, causal and linear time invariant (LTI) difference equation, the output $y(n)$ (pass-by response) at discrete time $n$ is computed from the present input $x(n)$ (sources) and the past output samples as

$$
y(n)=\sum_{i=0}^{M} b_{i}(n) x(n-i)-\sum_{j=1}^{N} a_{j}(n) y(n-j),
$$


where $M$ is the feedforward filter order and $N$ is the feedback filter order. Note that the IIR coefficients are changing at the source audio sample rate.

The final step in the synthesis is the inclusion of the Doppler effect as a propagation time delay, which can be inferred from the slope of the all-pass excess phase (refer to Figure 1). Each source-microphone path is characterized by a propagation time delay. A single time delay curve is obtained for the vehicle by averaging over all its source propagation time delay curves. The averaged time delay curve is linearly interpolated at the audio sample rate level before being applied to the time-varying implementation output.

\section{Automotive example}

The PBN synthesis is applied to an automotive indoor PBN measurement example. An energetic ASQ approach was used to identify and separate the source contributions from each component. In this example, six main components were identified and each one was characterized as consisting of a given number of sources. A linear array of microphones $7.5 \mathrm{~m}$ away from the nominal vehicle center line is employed to record the target responses and the propagated NTFs. The target microphones and near-field indicators near components were measured synchronously at the audio sampling frequency during an operational test on the chassis dyno.

A comparison of NTFs vs. IIR filters for a single source and two target microphone positions is considered. This comparison is done to evaluate the ability of the IIR filter to reproduce the sound pressure levels (SPL) of the NTF in a static scenario. Figure 2 shows the frequency response function of the measured NTF and the estimated $16^{\text {th }}$ order IIR filter for the two selected positions.

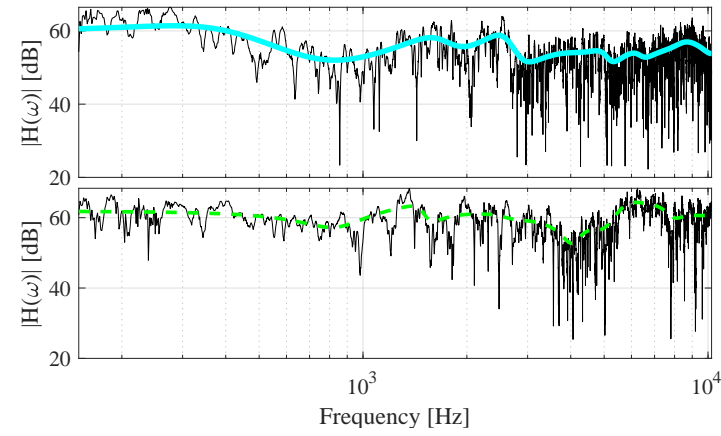

(a)

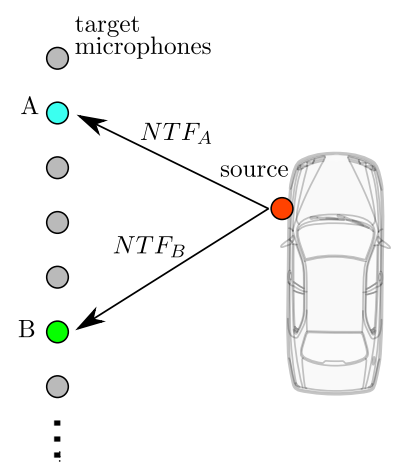

(b)

Fig. 2: (a) Frequency response function of the measured NTF (-) and the estimated $16^{\text {th }}$ order IIR filter at two target microphone positions: position A $(-)$ and position B $(=-=)$. 
Figure 2 shows that the IIR filters capture the main trends of the NTFs. Improvements in the fit can be achieved by increasing the filter order at the expense of instabilities and increase in computational time. Figure 3 shows the SPL calculated reciprocally in the center of the array from a moving source by applying a time-varying IIR filter. The SPL calculated at two target microphone positions with both the NTF (FIR) and IIR filters are also shown for reference.

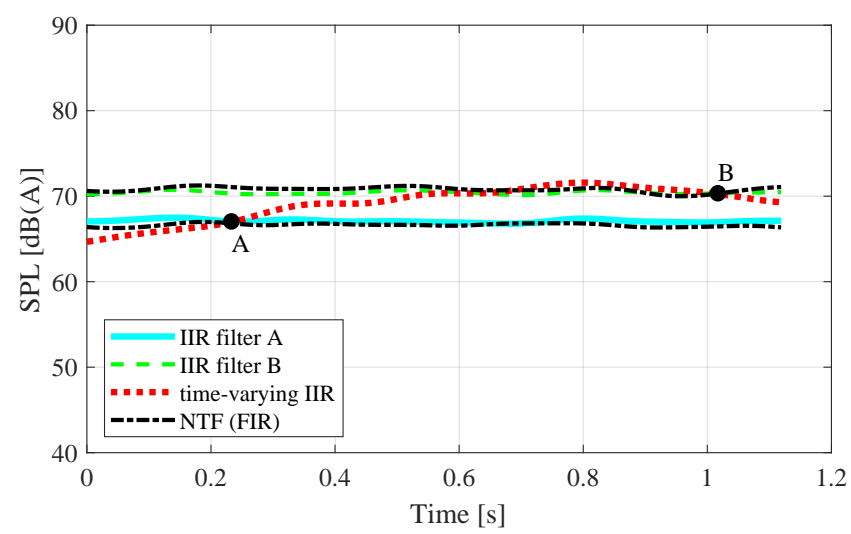

Fig. 3: (Color online) Equivalent SPL estimated at the center of the array from a moving source, by applying a time-varying IIR compared to the SPL from IIR and NTF (FIR) filters at two target microphone positions.

It can be noticed that the SPL from the IIR filter at both fixed positions are in agreement with the SPL from the NTFs. Moreover, the time-varying SPL value matches the respective target microphone position at the instants where they are being crossed by the vehicle.

From an indoor PBN test, figure 4 shows the SPL derived with the help of the time-varying IIR filter of each component contribution, as well as the summed (total) contribution, the measured overall level and the energetic TPA results from Simcenter Testlab as references. The measured overall level (OAL) provides a reference value for the time-varying filter and it is obtained by energetic superposition of the target microphone signals. Figure 4 demonstrates that the total TPA contribution derived from the IIR implementation, the measured OAL and the energetic TPA result are in agreement with each other. At the component level, the time-varying IIR matches with the energetic TPA, except for the central position of components 4 and 5 . The proper match of SPL demonstrates the feasibility of using IIR filters for PBN sound synthesis. Additionally, the time-varying IIR filter implementation yields, in a computationally fast manner, the PBN time signals which allow for complementary listening tests and sound quality assessment. 


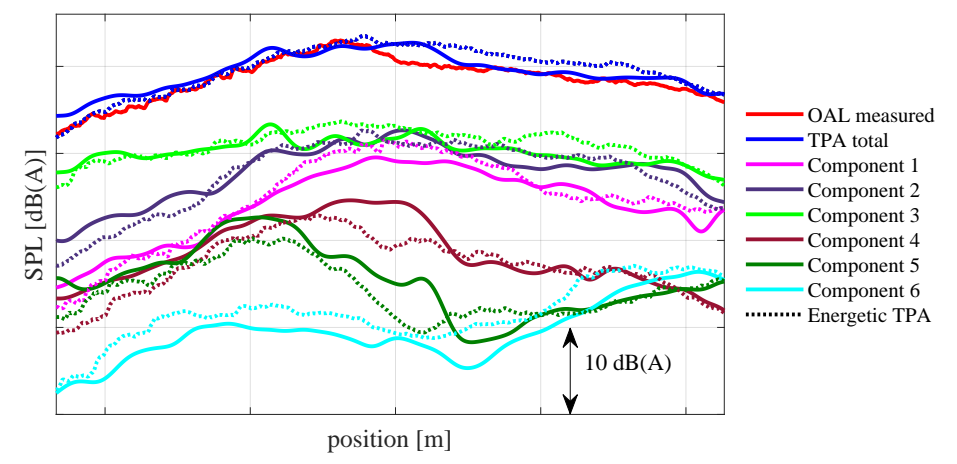

Fig. 4: (Color online) SPL of time-varying IIR filter for each component contribution, the total contribution, the measured overall level (OAL) and the energetic TPA from Simcenter Testlab (…...) at their respective component color scheme.

\section{Conclusions}

In this paper we presented a formulation for the sound synthesis of pass-by noise from a TPA approach. We validated the methodology in an automotive example, first at a single source and two target microphones and later in time-varying scenarios. The new approach was shown to match the SPL from Simcenter Testlab TPA while additionally providing the synthesized pass-by noise audio. Next step consist of validation of the synthesized audio against an exterior PBN test.

\section{Acknowledgements}

We gratefully acknowledge the European Commission for its support of the Marie Sklodowska Curie program through the H2020 ETN PBNv2 project (GA $721615)$.

\section{References}

1. M. Matsumoto, M. Tohyama, H. Yanagawa, Acoustical Science and Technology 24(5), 284 (2003). DOI 10.1250/ast.24.284

2. K. Janssens, P. Aarnoutse, P. Gajdatsy, L. Britte, F. Deblauwe, H. Van der Auweraer, in SAE 2011 Noise and Vibration Conference and Exhibition (2011). DOI 10.4271/2011-01-1609

3. B. Verrecas, P.V. de Ponseele, L. Britte, M. Roudey, P. Converset, M. Fréjacques, in Automotive Acoustics Conference 2nd Int. ATZ-Conference (2013), p. 19

4. P. Corbeels, in Aachen Acoustics Colloquium - AAC (2017)

5. J.M. Jot, V. Larcher, O. Warusfel, in Audio Engineering Society Convention 98 (Audio Engineering Society, 1995)

6. K. Janssens, F. Bianciardi, L. Britte, P.V. de Ponseele, in ISMA (2014), p. 18

7. J.O. Smith, Introduction to Digital Filters: With Audio Applications (Julius Smith, 2007) 